\title{
Electronic Identification of the Parental Phases and Mesoscopic Phase Separation of $\mathrm{K}_{x} \mathrm{Fe}_{2}-\mathrm{Se}_{2}$ Superconductors
}

\author{
F. Chen, ${ }^{1}$ M. Xu, ${ }^{1}$ Q. Q. Ge, ${ }^{1}$ Y. Zhang, ${ }^{1, *}$ Z. R. Ye, ${ }^{1}$ L.X. Yang, ${ }^{1}$ Juan Jiang, ${ }^{1}$ B. P. Xie, ${ }^{1}$ R. C. Che,${ }^{2}$ M. Zhang, ${ }^{3}$ \\ A. F. Wang, ${ }^{3}$ X. H. Chen, ${ }^{3}$ D. W. Shen, ${ }^{4}$ J.P. Hu, ${ }^{5}$ and D. L. Feng ${ }^{1, \dagger}$ \\ ${ }^{1}$ State Key Laboratory of Surface Physics, Department of Physics, and Advanced Materials Laboratory, Fudan University, \\ Shanghai 200433, People's Republic of China \\ ${ }^{2}$ Department of Materials Science and Advanced Materials Laboratory, Fudan University, \\ Shanghai 200433, People's Republic of China \\ ${ }^{3}$ Hefei National Laboratory for Physical Sciences at Microscale and Department of Physics, \\ University of Science and Technology of China, Hefei, Anhui 230026, People's Republic of China \\ ${ }^{4}$ State Key Laboratory of Functional Materials for Informatics, Shanghai Institute of Microsystem and Information Technology, \\ Chinese Academy of Sciences, Shanghai 20005, People's Republic of China \\ ${ }^{5}$ Department of Physics, Purdue University, West Lafayette, Indiana 47907, USA
}

(Received 27 July 2011; published 16 December 2011)

\begin{abstract}
The nature of the parent compound of a high-temperature superconductor (HTS) often plays a pivotal role in determining its superconductivity. The parent compounds of the cuprate HTSs are antiferromagnetically ordered Mott insulators, while those of the iron-pnictide HTSs are metals with spin-density-wave order. Here we report the electronic identification of two insulating parental phases and one semiconducting parental phase of the newly discovered family of $\mathrm{K}_{x} \mathrm{Fe}_{2-y} \mathrm{Se}_{2}$ superconductors. The two insulating phases exhibit Mott-insulator-like signatures, and one of the insulating phases is even present in the superconducting and semiconducting $\mathrm{K}_{x} \mathrm{Fe}_{2-y} \mathrm{Se}_{2}$ compounds. However, it is mesoscopically phaseseparated from the superconducting or semiconducting phase. Moreover, we find that both the superconducting and semiconducting phases are free of the magnetic and vacancy orders present in the insulating phases, and that the electronic structure of the superconducting phase could be developed by doping the semiconducting phase with electrons. The rich electronic properties discovered in these parental phases of the $\mathrm{K}_{x} \mathrm{Fe}_{2-y} \mathrm{Se}_{2}$ superconductors provide the foundation for studying the anomalous behavior in this new class of iron-based superconductors.
\end{abstract}

DOI: 10.1103/PhysRevX.1.021020

\section{INTRODUCTION}

The superconductivity in high-temperature superconductors (HTSs) often arises from the doping of their parent compounds. While the parent compounds for the cuprate HTSs are antiferromagnetic Mott insulators [1], those for the iron-pnictide HTSs are metals with spin-density-wave order [2,3], highlighting the differences between these two families of HTSs. $A_{x} \mathrm{Fe}_{2-y} \mathrm{Se}_{2}(A=\mathrm{K}, \mathrm{Cs}, \mathrm{Rb}, \ldots)$ is a new series of iron-based superconductors discovered recently, whose peculiar properties have generated a lot of interest $[4,5]$. For example, angle-resolved-photoemissionspectroscopy (ARPES) experiments did not observe any hole Fermi surface near the center of the Brillouin zone of $\mathrm{K}_{x} \mathrm{Fe}_{2-y} \mathrm{Se}_{2}$, thus ruling out the presence of the common $\mathrm{s}^{ \pm}$-pairing symmetry found in other iron-based HTSs [6].

\footnotetext{
*yanzhangfd@fudan.edu.cn
}

†dlfeng@fudan.edu.cn

Published by the American Physical Society under the terms of the Creative Commons Attribution 3.0 License. Further distribution of this work must maintain attribution to the author(s) and the published article's title, journal citation, and DOI.
Moreover, it appears that the superconducting phase is not adjacent to any metallic spin-density-wave state in the phase diagram, but rather, it is on the border of an insulating and magnetically ordered state accompanied by an iron-vacancy order $[7,8]$. Neutron-scattering studies found that the ordered magnetic moment could be as large as $3.3 \mu_{B}$ per iron cation [9], which is the largest among all the ferropnictides and ferrochalcogenides discovered so far. Further thermal-power and transmission-electronmicroscope (TEM) measurements were able to distinguish two antiferromagnetic insulating (AFI) phases of $\mathrm{K}_{x} \mathrm{Fe}_{2-y} \mathrm{Se}_{2}$ : an "AFI1" phase, characterized by a positive thermal power and a superlattice-modulation wave vector $(1 / 5,3 / 5,0)$, indexed using the tetragonal unit cell with lattice parameters of $a=3.913 \AA$ and $c=14.10 \AA$ and a space group I4/mmm, and an "AFI2" phase, characterized by a negative thermal power and a superlattice-modulation wave vector $(1 / 4,3 / 4,0)[7,10]$. Theoretically, these antiferromagnetic insulating compounds were proposed to be the parent compounds for the $\mathrm{A}_{x} \mathrm{Fe}_{2-y} \mathrm{Se}_{2}$ superconductor $[11,12]$. Since the iron-vacancy order was shown to cause a band narrowing for ferrochalcogenides and drive the system into a Mott-insulator phase, this observation invokes 
an intriguing possibility that the iron-based HTSs could also be viewed as doped Mott insulators, and thus there could even be a unifying mechanism for both the copperbased and the iron-based HTSs.

Here we report electronic-structure-based identification of two insulating phases and one semiconducting phase of $\mathrm{K}_{x} \mathrm{Fe}_{2-y} \mathrm{Se}_{2}$ by angle-resolved photoemission spectroscopy. We have found compelling evidence for the presence of Mott-like physics in the insulating phases. Moreover, one of the insulating phases is present in the semiconducting and superconducting compounds, but phase-separated at a mesoscopic scale from the semiconducting and superconducting phases. This mesoscopicscale phase separation is most likely the cause of the complications seen in various earlier measurements [9,13-15]. No band folding that corresponds to the magnetic and vacancy orders in the insulating phases is observed in the electronic structures of the semiconducting and superconducting phases. Furthermore, we show that the semiconducting phase could become the superconducting one upon electron doping. The rich electronic structures discovered in these parental phases highlight the complexity and instabilities in $\mathrm{K}_{x} \mathrm{Fe}_{2-y} \mathrm{Se}_{2}$ and provide a starting point for resolving the mystery of the highly unconventional superconductivity in this new series of iron-based chalcogenides.

\section{EXPERIMENTAL PROCEDURES}

$\mathrm{K}_{x} \mathrm{Fe}_{2-y} \mathrm{Se}_{2}$ single crystals were synthesized by the selfflux method as described elsewhere in detail [7]; they show flat, shiny, dark-black surfaces. The superconducting sample shows an onset superconducting-transition temperature $\left(T_{\mathrm{c}}\right)$ of $31.7 \mathrm{~K}$, and it reaches zero resistivity at $31.2 \mathrm{~K}$. The actual chemical compositions of the samples under study were determined by energy-dispersive $\mathrm{x}$-ray spectroscopy, which gives $\mathrm{K}_{0.77} \mathrm{Fe}_{1.65} \mathrm{Se}_{2}$ for the superconductor, $\mathrm{K}_{0.65} \mathrm{Fe}_{1.67} \mathrm{Se}_{2}$ for the semiconductor, $\mathrm{K}_{0.78} \mathrm{Fe}_{1.59} \mathrm{Se}_{2}$ for the AFI1, and $\mathrm{K}_{0.95} \mathrm{Fe}_{1.61} \mathrm{Se}_{2}$ for the AFI2, respectively. The in-house ARPES measurements were performed at Fudan University with $21.2 \mathrm{eV} \mathrm{He}-\mathrm{I} \alpha$ light from a SPECS UV-light-source discharge lamp. The synchrotron ARPES experiments were performed at the Beamline 5-4 station of the Stanford Synchrotron Radiation Lightsource (SSRL) facility. All the data were taken with Scienta electron analyzers, the overall energy resolution is $15 \mathrm{meV}$ in-house or $10 \mathrm{meV}$ at SSRL, and angular resolution is $0.3^{\circ}$. The samples were cleaved in situ, and measured under ultrahigh vacuum of $5 \times 10^{-11}$ torr units of pressure. High-resolution transmission-electron-microscopy (HRTEM) images and selective-area electron-diffraction patterns were obtained at room temperature, using a JEOL JEM-2100F transmissionelectron microscope equipped with a postcolumn Gatan imaging filter (GIF-Tridium) at an acceleration voltage of $200 \mathrm{kV}$.
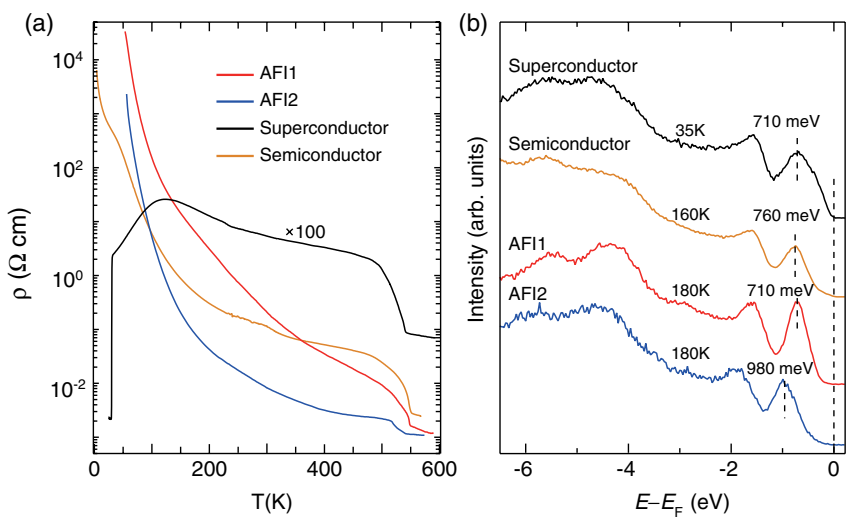

FIG. 1. Resistivities and valence bands of various $\mathrm{K}_{x} \mathrm{Fe}_{2-y} \mathrm{Se}_{2}$ compounds. (a) In-plane resistivities as a function of temperature for the AFI1 and AFI2 insulating compounds, the semiconducting compound, and the superconducting compound with the superconducting-transition temperature $\left(T_{\mathrm{c}}\right)$ of $31 \mathrm{~K}$. The resistivity of the superconducting sample is multiplied by 100 . (b) Valence-band photoemission spectra at the $\Gamma$ point for the insulators, semiconductor, and superconductor. The dashed vertical lines are guides to eyes for the peak positions of the feature near $-1 \mathrm{eV}$. If not specified otherwise, data here and hereafter were taken with $21.2 \mathrm{eV}$ photons from an in-house helium-discharge lamp. The data for superconductor, semiconductor, and insulators were measured at 35, 160, and $180 \mathrm{~K}$, respectively.

\section{PHASE SEPARATION IN SUPERCONDUCTING AND SEMICONDUCTING $\mathrm{K}_{x} \mathrm{Fe}_{2-y} \mathrm{Se}_{2}$}

To reveal the nature of the parent compound of this new family of iron-based superconductors, we have studied with ARPES the series of $\mathrm{K}_{x} \mathrm{Fe}_{2-y} \mathrm{Se}_{2}$ compounds listed in the previous section. As illustrated in Fig. 1(a), the resistivity behaviors of these $\mathrm{K}_{x} \mathrm{Fe}_{2-y} \mathrm{Se}_{2}$ samples are rather versatile, including the insulating and superconducting ones [7], and a semiconducting one that is much less insulating below $100 \mathrm{~K}$ as reported before [13]. Interestingly, the resistivity of the superconductor exhibits an insulating behavior above $120 \mathrm{~K}$, while that of the semiconducting compound exhibits an inflexion at $30 \sim 50 \mathrm{~K}$. Moreover, the resistivity anomaly near $550 \mathrm{~K}$ is present in all the samples, indicating the formation of the vacancy order [7,14]. Figure 1(b) shows the valence-bandphotoemission spectra near the zone center $(\Gamma)$ over a large energy window for all four types of compounds. The main spectral features are comparable in all cases; however, difference does show up quantitatively. For example, the feature near $-1 \mathrm{eV}$ differs by $270 \mathrm{meV}$ for AFI1 and AFI2, indicating that these two insulating phases are indeed electronically different. The peak of the semiconducting sample is situated between AFI1 and AFI2, while the peak position of the superconducting sample is the same as that of AFI1, but it clearly contains a shoulder structure near the Fermi energy $\left(E_{\mathrm{F}}\right)$. 

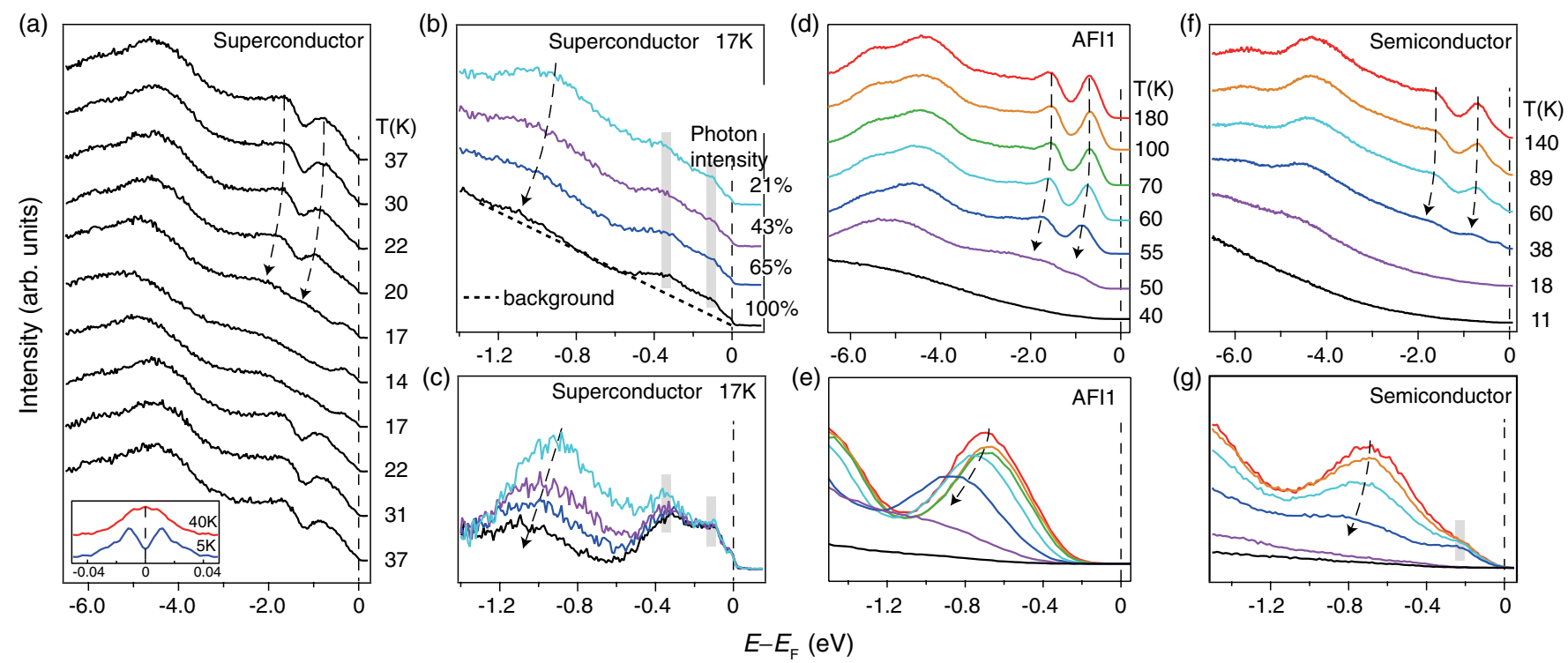

FIG. 2. Photoemission charging effects in $\mathrm{K}_{x} \mathrm{Fe}_{2-y} \mathrm{Se}_{2}$. (a) Temperature dependence of the photoemission-energy-distribution curve (EDC) at the $\Gamma$ point of the superconductor. The inset is the symmetrized EDCs across $T_{c}$, illustrating a superconducting gap of about $10 \mathrm{meV}$ at the electron-Fermi surface around the zone corner. (b) EDC at the $\Gamma$ point of the superconductor as a function of the photon intensity. Data were taken at $17 \mathrm{~K}$. (c) The EDCs after deducting the background [diagonal short-dashed line in (b)] to highlight the peak position shift due to the charging. (d) Temperature dependence of the EDCs at the $\Gamma$ point for the AFI1 sample. (e) The lowenergy portion of data in (d). (f) and (g) are the same as (d) and (e), respectively, but for the semiconducting sample. The arrows on the dashed curves in all panels indicate the band shift with enhanced charging effects. The shaded bars in (b), (c), and (g) indicate charging-free features.

The rather similar valence bands in Fig. 1(b) raise a question as to whether these features are related to the intrinsic electronic structures of the drastically different phases of $\mathrm{K}_{x} \mathrm{Fe}_{2-y} \mathrm{Se}_{2}$. The detailed temperature dependence in Fig. 2(a) shows that the two features at -1.6 and $-0.7 \mathrm{eV}$ in the superconducting sample fade away at $17 \mathrm{~K}$ and disappear at $14 \mathrm{~K}$. The features reappear upon increasing the temperature. Moreover, if we reduce the intensity of the $21.2 \mathrm{eV}$ photons, these valence-band features recover [Fig. 2(b)]. As shown in the background-deducted spectra in Fig. 2(c), the valence band moves toward $E_{\mathrm{F}}$ upon decreasing the incident-photon intensity. The reaction of the valence-band peaks at -1.6 and $-0.7 \mathrm{eV}$ to the photon intensity and temperature are the typical charging behaviors of an insulator, since the resistivity quickly increases at lower temperatures. When the photoelectrons leave an insulating region, the electric charge and potential will build up, which would smear and shift the photoemission spectrum to higher binding energies [Fig. 2(b)]. Evidently, as the number of photoelectrons is decreased by reducing the photon intensity, the charging behavior could be suppressed [Fig. 2(c)]. Therefore, the charging features most probably originate from some insulating regions in the material. Those insulating regions are most likely of the AFI1 type, judging both from their valence-band positions in Fig. 1(b) and from TEM evidence presented later in Fig. 5. On the other hand, the low-energy features in the first $0.5 \mathrm{eV}$ below $E_{\mathrm{F}}$ show charging-free behavior in
Fig. 2(c) and are absent in the AFI1 sample [Figs. 2(d) and 2(e)]. These observations indicate that the low-energy features belong to the metallic regions that are phaseseparated from the insulating regions. In fact, the inset of Fig. 2(a) shows that these metallic states open a superconducting gap near $E_{\mathrm{F}}$ below $T_{\mathrm{c}}$ [6]. Furthermore, the line shape in the first $0.2 \mathrm{eV}$ below $E_{\mathrm{F}}$ is independent of the charging potential developed in the insulating regions, which proves that the electric fields in the insulating regions are well screened by the boundary charges of the metallic regions and thus do not affect the photoemission there. Similar phase separation in the semiconducting sample is illustrated in Figs. 2(f) and 2(g). The insulating feature (below $-0.5 \mathrm{eV}$ ) already shows charging behavior at temperatures higher than $60 \mathrm{~K}$, while the semiconducting features (between $-0.5 \mathrm{eV}$ and $E_{\mathrm{F}}$ ) just start to show some charging behavior below $38 \mathrm{~K}$, as expected from the increased resistivity of the semiconductor. Moreover, while the positions of the insulating features in the semiconducting and superconducting compounds are almost the same as those in AFI1 at high temperatures, suggesting that the features originate from the same AFI1 insulating phase, the charging behavior in the superconducting compound happens at the much lower temperature of $20 \mathrm{~K}$. This could be attributed to the variations of insulating-region size in different compounds. For the superconductor, the phase separation should happen in a mesoscopic scale, in light of the vacancy-ordered or 
vacancy-disordered domains observed in previous TEM studies [10]. Indeed, as will be discussed in Sec. VI, the phase separation between the superconducting and semiconducting regions and the AFI1 regions at the scales of several nanometers could be further demonstrated by our TEM measurements on the superconducting and semiconducting $\mathrm{K}_{x} \mathrm{Fe}_{2-y} \mathrm{Se}_{2}$ compounds.

\section{THE MOTT-LIKE PHYSICS IN INSULATING $\mathrm{K}_{x} \mathrm{Fe}_{2-y} \mathrm{Se}_{2}$}

The prominent contribution of the insulating phase to the valence band suggests that the insulating phase makes up a rather large fraction of the semiconducting and superconducting samples. Figure 3 compares the electronic structures of the insulating compounds with those of the superconductor measured at $11 \mathrm{~K}$, so that the contribution to the spectra from the insulating regions in the superconductor is minimized by their own charging. The lowenergy spectral weight in the superconducting case seems to be transferred into the feature around $-0.7 \mathrm{eV}$ in Figs. 3(a) and 3(b) for AFI1, and $-1 \mathrm{eV}$ in Figs. 3(c) and 3(d) for AFI2. Such spectral-weight transfer behaviors resemble the opening of the Mott-Hubbard gap when the Coulomb interactions between the electrons are turned on $[1,11,12]$. Such Mott-like behavior in the insulating phases has recently been predicted in LSDA+U (local-spindensity approximation plus Coulomb interactions) calculations for both AFI1 and AFI2 [16-18]. It was shown that the vacancy order alone would not open a large energy gap

(a)

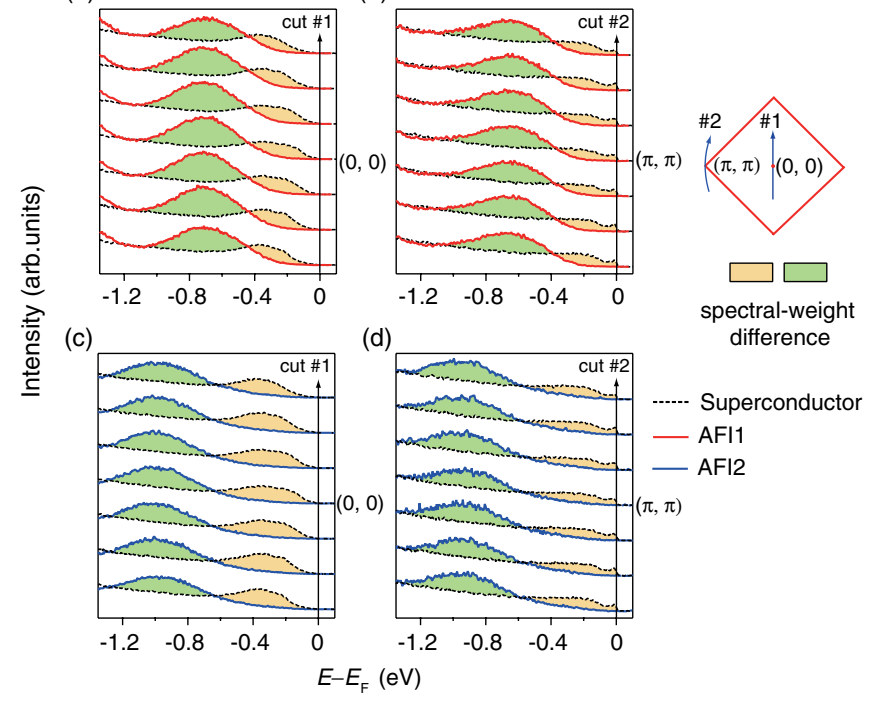

FIG. 3. Spectral-weight difference between the insulators and the superconductor. (a) and (b) compare EDCs of the AFI1 insulator and the superconductor along cuts \#1 and \#2 across the projected two-dimensional Brillouin-zone center and corner, respectively. (c) and (d) make the same comparison between AFI2 and the superconductor. Data for the insulators and superconductor were taken at 180 and $11 \mathrm{~K}$, respectively. in the band structure [16-19]. When the magnetic order was included, the band was renormalized greatly. A gap would open for AFI1 even without including the Coulomb interactions in the calculation as the magnetic order was extremely strong there, but including the interactions would further increase this gap $[16,17]$, while the interactions were needed for the AFI2 case to open a gap [18]. Our observation of the gap in both cases, particularly in the AFI2 case, suggests that the Coulomb interactions are an important factor of the physics here. The essentially nondispersive feature of the insulators in Fig. 3 further agrees with these calculations.

\section{COMPARISON OF SUPERCONDUCTING AND SEMICONDUCTING $\mathrm{K}_{x} \mathrm{Fe}_{2-y} \mathrm{Se}_{2}$}

In contrast to the drastic difference between the electronic structures of the insulating and superconducting phases, the low-energy features observed in the semiconducting and superconducting samples show similar charging-free behavior. This result suggests an intimate relationship between these semiconducting and superconducting phases. Figure 4 compares their low-energy electronic structures along two high-symmetry cuts in the threedimensional (3D) Brillouin zone [20]. As illustrated in Fig. 4(a) for the superconductor, there is a Fermi surface around the $\mathrm{Z}$ point that is contributed by a band named $\kappa$ and four Fermi cylinders around the zone edges that are contributed by a band named $\delta$. The $\delta$ band is in fact almost two-fold degenerate but not distinguishable here. These are all electronlike Fermi surfaces. Figures 4(c) and 4(d) show the photoemission intensity for the superconducting and semiconducting phases, respectively, along cut \#1 across $\Gamma$ as illustrated in Fig. 4(a). For the superconducting phase, an indirect gap of about $25 \mathrm{meV}$ could be observed between the top of the two almost-degenerate bands at $\Gamma$ called $\alpha$ and $\beta$ and the bottom of the $\delta$ band at the zone corner [Fig. 4(c)]. As shown in Fig. 4(d), the chemical potential of the semiconductor is coincidentally situated in this gap. Compared with the superconductor, the semiconducting sample shows that the top of the $\alpha$ and $\beta$ bands shifts up by $55 \mathrm{meV}$, and thus is $20 \mathrm{meV}$ below $E_{\mathrm{F}}$ at $\Gamma$. Along cut \#2 across $\mathrm{Z}$, the gap between $\alpha / \beta$ and $\kappa$ or $\delta$ is rather small as shown in Fig. 4(e) for the superconducting phase, but there is a gap of $20 \mathrm{meV}$ between the chemical potential and the top of the $\alpha$ and $\beta$ bands for the semiconducting phase [Fig. 4(f)]. Note that the $\delta$ and $\kappa$ bands are absent near $E_{\mathrm{F}}$ in the semiconducting samples. One could have deduced the unoccupied $\delta$ bands to be the dashed curves in Fig. 4(b), if it had shifted the same amount as the $\beta$ band at the zone corner. However, no traces of bands above $E_{\mathrm{F}}$ in the semiconducting sample were detectable up to $100 \mathrm{~K}$, which means that the unoccupied states are too far to be populated by the temperature broadening of the Fermi-Dirac distribution [21]. Therefore, we could safely deduce a lower limit of the band gap of $40 \mathrm{meV}$ in the semiconducting sample 
(a)

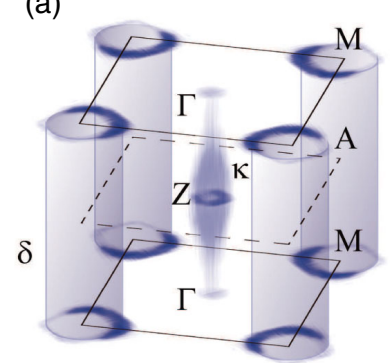

(c) Superconductor

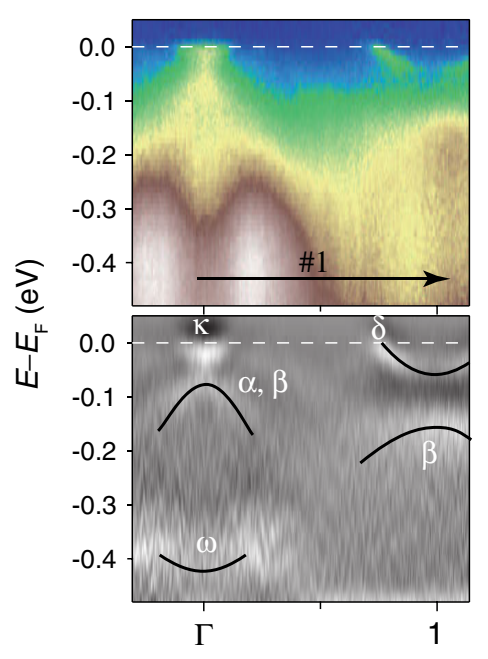

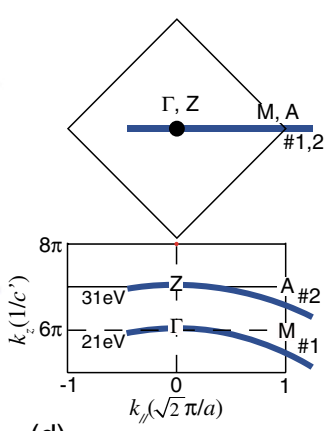

(d)

(b)

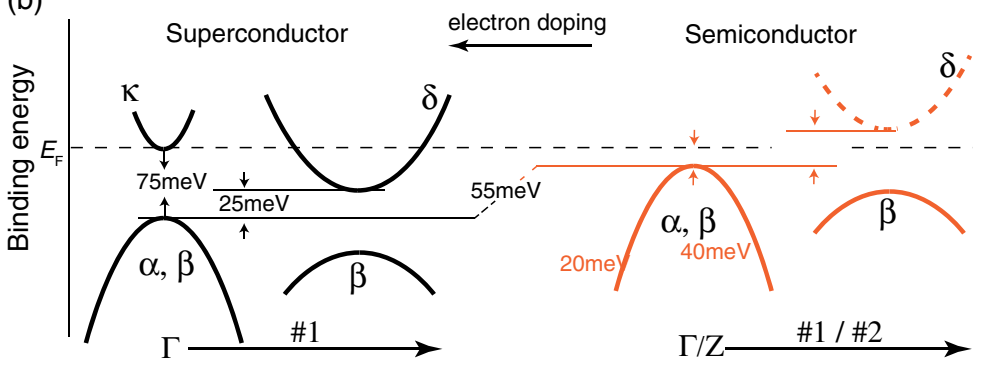

(e)

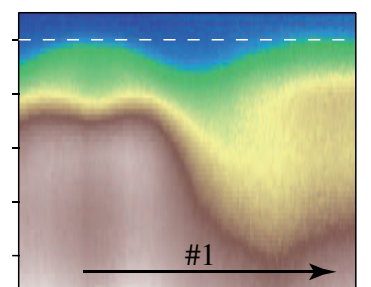

Serperconductor

(f)
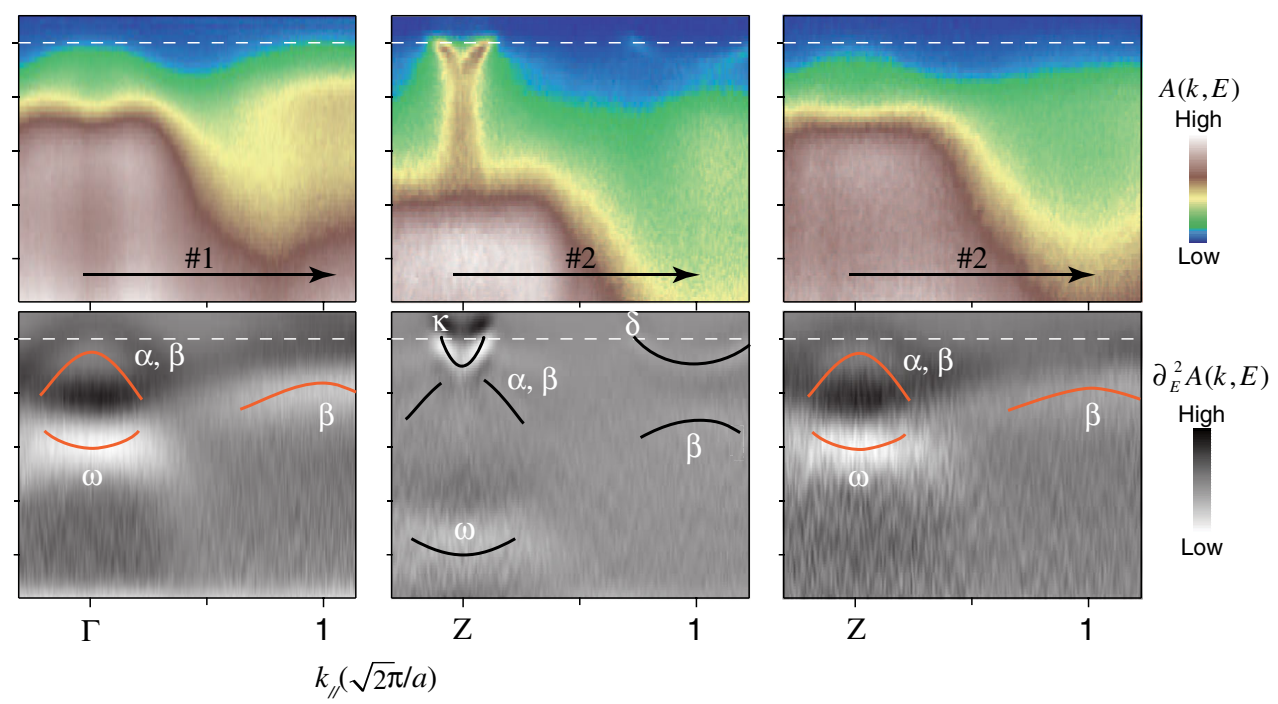

FIG. 4. Low-energy electronic structures of the superconducting and semiconducting $\mathrm{K}_{x} \mathrm{Fe}_{2-y} \mathrm{Se}_{2}$. (a) Left: the Fermi surface of the superconducting phase in the 3D Brillouin zone. Right: the two-momentum cut \#1 and cut \#2 sampled with $21 \mathrm{eV}$ and $31 \mathrm{eV}$ photons, respectively, are illustrated in the two-dimensional cross-sections of the 3D Brillouin zone. $c^{\prime}$ is the distance between neighboring FeAs layers, and $a$ is the Fe-Fe distance in the FeAs plane. (b) A sketch of the band-structure evolution from the semiconductor to the superconductor. (c) The photoemission intensities (upper panel) and their second derivatives with respect to energy (lower panel) along cut \#1 across $\Gamma$ for the superconductor taken at $35 \mathrm{~K}$. (d) is the same as (c) but for the semiconductor taken at $100 \mathrm{~K}$. (e) and (f) are same as (c), (d), respectively, except the data were taken along cut \#2 across Z.

[Fig. 4(b)], which is consistent with the small indirect gap of about $30 \mathrm{meV}$ deduced from the optical-conductivity data of the semiconducting $\mathrm{K}_{0.8} \mathrm{Fe}_{2-y} \mathrm{Se}_{2}$ [13].

The electronic structures of the superconducting and semiconducting phases are summarized in Fig. 4(b). Our results suggest that, because they have similar electronic structures, the semiconducting phase is perhaps a closer parent compound to the $\mathrm{K}_{x} \mathrm{Fe}_{2-y} \mathrm{Se}_{2}$ superconductor than the insulators are. With electron doping, the semiconductor might evolve into a superconductor. Electronically, the semiconducting phase of $\mathrm{K}_{x} \mathrm{Fe}_{2-y} \mathrm{Se}_{2}$ appears like a conventional semiconductor with fully occupied bands and a small band gap and no magnetic order, as discussed below. However, there is still nonrigid band behavior that suggests possible correlation effects involved. For example, the $\omega$ and $\kappa$ bands shift up much more than the $\alpha$ and $\beta$ bands in the semiconductor compared with their positions in the superconductor. The $\omega$ band shifts up by about $200 \mathrm{meV}$, and the bottom of the $\kappa$ band is now far from the top of the $\alpha$ and $\beta$ bands along cut \#2 in the semiconductor. Such an interesting semiconducting phase has largely been neglected in previous theoretical and experimental studies. Our findings identify it as a possible starting point for modeling the superconductivity in $\mathrm{K}_{x} \mathrm{Fe}_{2-y} \mathrm{Se}_{2}$, which is rather unique compared with other HTSs.

\section{DISCUSSION AND CONCLUSION}

We note that the earlier TEM studies have found that both a vacancy-ordered phase and a vacancy-disordered phase exist in different regions of the sample [10]. It was speculated that the vacancy-ordered phase was insulating, while the vacancy-disordered phase was superconducting. Consistently, two-step magnetic penetration [22] and sharp nuclear-magnetic-resonance (NMR) spectra [23] observed in $\mathrm{K}_{x} \mathrm{Fe}_{2-y} \mathrm{Se}_{2}$ suggest that the superconducting phase does not coexist with magnetic order. On the other hand, neutron-scattering, Mössbauer-spectroscopy, 
muon-spin-rotation-and-relaxation, and transport studies have suggested that the superconductivity coexists with the antiferromagnetic order, although each four-iron spin block even has a total moment as large as $13 \mu_{B}$ $[9,14,15,24]$. Surprisingly, one recent TEM study found that the diffraction peaks corresponding to the vacancy order diminished when the system entered the superconducting state at low temperatures, suggesting that the vacancy order disappeared [25]. This is hard to reconcile with the large energy scale involved for the vacancy order, but it seems to provide a natural explanation for the NMR data. Thus, to date, there are still controversies and unsolved puzzles about whether the magnetic order and the superconductivity coexist in $\mathrm{K}_{x} \mathrm{Fe}_{2-y} \mathrm{Se}_{2}$.

The charging behavior presented in Fig. 2 indicates that the superconducting $\mathrm{K}_{x} \mathrm{Fe}_{2-y} \mathrm{Se}_{2}$ compound is composed of superconducting regions and insulating regions, and the semiconducting $\mathrm{K}_{x} \mathrm{Fe}_{2-y} \mathrm{Se}_{2}$ compound is composed of semiconducting regions and insulating regions. The lowenergy electronic structure of the superconducting and semiconducting regions does not exhibit any folded feature related to the strong vacancy or magnetic order in Fig. 4, despite clear diffraction patterns for the $\sqrt{5} \times \sqrt{5}$ vacancy order that have been observed with TEM in both the superconducting and the semiconducting samples [Fig. 5(a)]. Therefore, the superconducting or semiconducting regions should be free of these orders. Consequently, the insulating regions in these compounds should be the AFI1 phase, where the magnetic and $\sqrt{5} \times \sqrt{5}$ vacancy orders are strongly tied with each other [26]. The superstructure Bragg peaks related to the vacancy order of the AFI2 phase were not observed, which indicates that the AFI2 phase might be chemically less stable than AFI1 in the composition range of the superconductor and semiconductor. Since the charging in the superconductor and semiconductor occurs at a much lower temperature than in the insulator, their insulating domains must be microscopic. To illustrate the length scale of such a chemical phase separation, we have taken HRTEM images of the semiconducting and superconducting samples, as shown in Figs. 5(b) and 5(c), which appear to have certain domain structures. To further visualize the vacancy-ordered and vacancydisordered regions [10], a fast Fourier transform (FFT) is conducted on the HRTEM images in Figs. 5(b) and 5(c), and subsequently another inverse-FFT is conducted on the intensities only around the $( \pm 1 / 5, \pm 3 / 5,0)$ superstructure spots. In this way, we could obtain the effectivedark-field image of the samples in Figs. 5(d) and 5(e) respectively. Here, the image contrast directly reflects the strength of the vacancy order, and the mesoscopic phase separation at the scale of several nanometers is visualized: Some regions exhibit rather strong $\sqrt{5} \times \sqrt{5}$-ordered structure, while other regions exhibit rather weak order [27]. The average domain size of the semiconductor is bigger than that of the superconductor. We note that the bright regions are the AFI1 phase with strong order; however, the weak $\sqrt{5} \times \sqrt{5}$ order in the dark regions does not mean that these superconducting and semiconducting regions contain
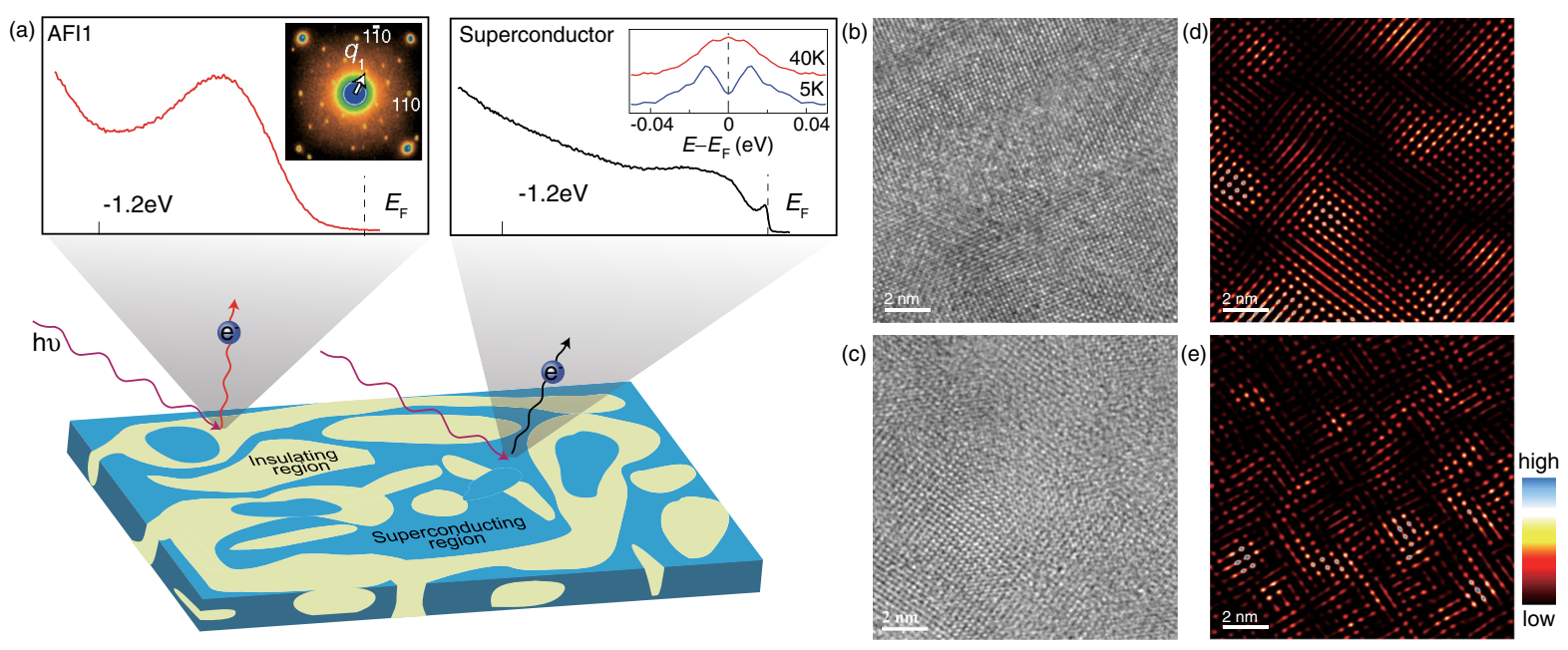

FIG. 5. (a) Cartoon for mesoscopic phase separation in superconducting $\mathrm{K}_{x} \mathrm{Fe}_{2-y} \mathrm{Se}_{2}$. Different regions exhibit different photoemission-spectroscopic signatures. Inset in the left panel of (a): The diffraction pattern of the $\sqrt{5} \times \sqrt{5}$ order was observed with TEM in both the superconductors and semiconductors, indicating a mixing of either the superconducting or the semiconducting phase with the AFI1 phase. The arrow in the diffraction pattern indicates the superlattice-modulation wave vector $q_{1}=(1 / 5,3 / 5,0)$. The TEM data was collected at room temperature. Inset in the right panel of (a): The symmetrized EDCs of the superconductor across $T_{\mathrm{c}}$, illustrating a superconducting gap. (b) and (c) The typical raw HRTEM image of the semiconducting sample and superconducting sample, respectively. (d) and (e) The effective-dark-field images constructed from the images in (b) and (c) by using the ( $\pm 1 / 5$, $\pm 3 / 5,0)$ superstructure spots as described in the text. In such dark-field images, only the $\sqrt{5} \times \sqrt{5}$ vacancy order contributes to the image contrast, and the mesoscopic phase separation of vacancy-ordered (brighter) and vacancy-disordered (darker) regions is clearly illustrated. 
the $\sqrt{5} \times \sqrt{5}$ order. It is partially a mathematical artefact because only the Bragg spots from the ordered regions were chosen to conduct the inverse-FFT and reconstruct the entire dark-field image. (A numerical simulation is presented in the supplementary material at Ref. [28] to illustrate this artefact.) Moreover, it has been shown that the ordered and disordered layers could be intercalated [10]; therefore, the vacancy-ordered regions in layers underneath the disordered regions could also contribute some intensity to the "dark" or weakly ordered region.

By considering the mesoscopic phase separation, one could resolve some of the aforementioned controversies related to these compounds. For example, the mysterious disappearance of the vacancy order at low temperatures in the recent TEM study of the superconductor might be explained by charging as well. That is, the Bragg peaks corresponding to the vacancy order could be smeared out by accumulated charges on the insulating domains, although the vacancy order did not disappear at low temperatures. Our findings further explain various anomalous properties of $\mathrm{K}_{x} \mathrm{Fe}_{2-y} \mathrm{Se}_{2}$, such as the very low chargecarrier density observed in the superconducting sample in optical studies [29]. Furthermore, it may also hint that the hump at $120 \mathrm{~K}$ in the resistivity of the superconductor and the inflexion around $30 \sim 50 \mathrm{~K}$ of the semiconductor are just a consequence of the competition between the metallic, semiconducting, and insulating regions in the same sample. In an earlier neutron-scattering study, a suppression of the ordered magnetic moment was found in the superconducting state and was taken as the evidence for the coexistence of the superconducting state and the magnetic order [9]. Based on the BCS theory of superconductivity, the superconducting coherence length $\xi=\hbar v_{F} / \pi \Delta$, where $\Delta$ is the superconducting gap. The coherence length of the $\mathrm{K}_{x} \mathrm{Fe}_{2-y} \mathrm{Se}_{2}$ superconductor is estimated to be about $1.2 \mathrm{~nm}$, comparable to the domain size. Therefore, the observed mesoscopic phase separation indicates that the suppression might be due to the proximity effect, as suggested in a recent theory [30].

All of the three insulating and semiconducting phases are chemically in the vicinity of the superconducting phase. The magnetically ordered insulating phases with a Mott-like gap may point out the importance of strong correlation for the superconductivity, while the clear band dispersion in the semiconducting phase may highlight the importance of itinerancy in the problem. We have shown that the electronic structure of the semiconducting phase resembles that of the superconducting phase more than those of the insulating phases do. However, it is also reasonable to speculate that the semiconducting phase might be developed from the insulating phases by varying their K, Fe, and Se compositions. Although the Fe vacancy order and magnetic order are destroyed in the semiconducting phase, spin fluctuations might still be present as in the case of a doped Mott insulator, and they could eventually be responsible for the emergence of the superconductivity upon further electron doping. In this regard, it is worthwhile to examine the magnetic properties of the semiconducting phase, for example, to check whether there are still local moments through neutron-scattering experiments.

To summarize, we have identified various phases of $\mathrm{K}_{x} \mathrm{Fe}_{2-y} \mathrm{Se}_{2}$ and provided electronic evidence for a mesoscopic phase separation of the superconducting and semiconducting phases and the AFI1 insulating phase. The spectral-weight transfer in the AFI1 and AFI2 phases resembles the opening of a Mott gap, indicating that the Coulomb interaction becomes evident when the magnetic order sets in. On the other hand, except for a strong renormalization of bandwidth, the electronic structures measured in the superconducting and semiconducting phases are not drastically altered from local-densityapproximation (LDA) results. The semiconducting phase is possibly a closer parent compound that may lead to the superconductor upon electron doping. Our results not only give a comprehensive understanding of various anomalous properties of this material, but they also provide the foundation for a microscopic understanding of this new class of iron-based high-temperature superconductors.

\section{ACKNOWLEDGMENTS}

We are grateful to Dr. Donghui Lu for experimental assistance at SSRL, and to Professor Ruibao Tao, Professor Maurice Rice, Professor Tao Xiang, Professor Nanlin Wang, and Professor Jianhui Dai for helpful discussions. Professor Jianqi Li kindly shared his unpublished preprint with us regarding his group's observation of the inplane phase separation by TEM. This work is supported in part by the National Science Foundation of China, Ministry of Education of China, Science and Technology Committee of Shanghai Municipal, and National Basic Research Program of China (973 Program) under Grants No. 2011CB921802 and No. 2011CBA00112. SSRL is operated by the US DOE, Office of Basic Energy Science, Divisions of Chemical Sciences and Material Sciences.

Note added.-A recent STM study on $\mathrm{K}_{x} \mathrm{Fe}_{2-y} \mathrm{Se}_{2}$ thin films observed clear evidence for the phase separation [31].

[1] P. A. Lee, N. Nagaosa, and X.-G. Wen, Doping a Mott Insulator: Physics of High-Temperature Superconductivity, Rev. Mod. Phys. 78, 17 (2006).

[2] Y. Kamihara, T. Watanabe, M. Hirano, and H. Hosono, Iron-Based Layered Superconductor $\mathrm{La}\left[\mathrm{O}_{1-x} \mathrm{~F}_{x}\right] \mathrm{FeAs}(x=$ 0.05-0.12) with $T_{\mathrm{c}}=26 \mathrm{~K}, \mathrm{~J}$. Am. Chem. Soc. 130, 3296 (2008).

[3] X. H. Chen, T. Wu, G. Wu, R. H. Liu, H. Chen, and D. F. Fang, Superconductivity at $43 \mathrm{~K}$ in $\mathrm{SmFeAsO}_{1-x} \mathrm{~F}_{x}$, Nature (London) 453, 761 (2008). 
[4] J. G. Guo, S. F. Jin, G. Wang, S. C. Wang, K. X. Zhu, T. T. Zhou, M. He, and X.L. Chen, Superconductivity in the Iron Selenide $\mathrm{K}_{x} \mathrm{Fe}_{2-y} \mathrm{Se}_{2}(0 \leq x \leq 1.0)$, Phys. Rev. B 82, 180520 (2010).

[5] J. J. Ying, X. F. Wang, X. G. Luo, A. F. Wang, M. Zhang, Y. J. Yan, Z. J. Xiang, R. H. Liu, P. Cheng, G. J. Ye, and $\mathrm{X}$. H. Chen, Superconductivity and Magnetic Properties of Single Crystals of $\mathrm{K}_{0.75} \mathrm{Fe}_{1.66} \mathrm{Se}_{2}$ and $\mathrm{Cs}_{0.81} \mathrm{Fe}_{1.61} \mathrm{Se}_{2}$, Phys. Rev. B 83, 212502 (2011).

[6] Y. Zhang, L. X. Yang, M. Xu, Z. R. Ye, F. Chen, C. He, H. C. Xu, J. Jiang, B. P. Xie, J. J. Ying, X. F. Wang, X. H. Chen, J. P. Hu, M. Matsunami, S. Kimura, and D. L. Feng, Nodeless Superconducting Gap in $\mathrm{A}_{x} \mathrm{Fe}_{2} \mathrm{Se}_{2}(\mathrm{~A}=\mathrm{K}, \mathrm{Cs})$ Revealed by Angle-Resolved Photoemission Spectroscopy, Nature Mater. 10, 273 (2011).

[7] Y. J. Yan, M. Zhang, A. F. Wang, J. J. Ying, Z. Y. Li, W. Qin, X.G. Luo, J.Q. Li, J.P. Hu, and X.H. Chen, Electronic and Magnetic Phase Diagram in $\mathrm{K}_{x} \mathrm{Fe}_{2-y} \mathrm{Se}_{2}$ Superconductors, arXiv:1104.4941.

[8] M. H. Fang, H. D. Wang, C. H. Dong, Z. J. Li, C. M. Feng, J. Chen, and H. Q. Yuan, Fe-Based Superconductivity with $T_{\mathrm{c}}=31 \mathrm{~K}$ Bordering an Antiferromagnetic Insulator in (Tl, K)Fe $\mathrm{Fe}_{x}$, Europhys. Lett. 94, 27009 (2011).

[9] W. Bao, Q. Huang, G. F. Chen, M. A. Green, D. M. Wang, J. B. He, X. Q. Wang, and Y. Qiu, A Novel Large Moment Antiferromagnetic Order in $\mathrm{K}_{0.8} \mathrm{Fe}_{1.6} \mathrm{Se}_{2}$ Superconductor, Chin. Phys. Lett. 28, 086104 (2011).

[10] Z. Wang, Y. J. Song, H. L. Shi, Z. W. Wang, Z. Chen, H. F. Tian, G. F. Chen, J. G. Guo, H.X. Yang, and J. Q. Li, Microstructure and Ordering of Iron Vacancies in the Superconductor System $\mathrm{K}_{y} \mathrm{Fe}_{x} \mathrm{Se}_{2}$ as Seen via Transmission Electron Microscopy, Phys. Rev. B 83, 140505 (2011).

[11] R. Yu, J.-X. Zhu, and Q. Si, Mott Transition in Modulated Lattices and Parent Insulator of $(\mathrm{K}, \mathrm{Tl})_{y} \mathrm{Fe}_{x} \mathrm{Se}_{2}$ Superconductors, Phys. Rev. Lett. 106, 186401 (2011).

[12] Y. Zhou, D. H. Xu, F. C. Zhang, and W. Q. Chen, Theory for Superconductivity in $(\mathrm{Tl}, \mathrm{K}) \mathrm{Fe}_{x} \mathrm{Se}_{2}$ as Doped Mott Insulator, Europhys. Lett. 95, 17003 (2011).

[13] Z. G. Chen, R. H. Yuan, T. Dong, G. Xu, Y. G. Shi, P. Zheng, J. L. Luo, J. G. Guo, X. L. Chen, and N. L. Wang, An Infrared Probe of the Electronic Structure of the New Iron Selenides $\mathrm{K}_{0.8} \mathrm{Fe}_{2-y} \mathrm{Se}_{2}$, Phys. Rev. B 83, 220507 (2011).

[14] R. H. Liu, X. G. Luo, M. Zhang, A. F. Wang, J. J. Ying, X. F. Wang, Y. J. Yan, Z. J. Xiang, P. Cheng, G. J. Ye, Z. Y. $\mathrm{Li}$, and X. H. Chen, Coexistence of Superconductivity and Antiferromagnetism in Single Crystals $\mathrm{A}_{0.8} \mathrm{Fe}_{2-y} \mathrm{Se}_{2}$ $(\mathrm{A}=\mathrm{K}, \quad R b, C s, \quad T l / K$ and $T l / R b):$ Evidence from Magnetization and Resistivity, Europhys. Lett. 94, 27008 (2011).

[15] Z. Shermadini, A. Krzton-Maziopa, M. Bendele, R. Khasanov, H. Luetkens, K. Conder, E. Pomjakushina, S. Weyeneth, V. Pomjakushin, O. Bossen, and A. Amato, Coexistence of Magnetism and Superconductivity in the Iron-Based Compound $\mathrm{Cs}_{0.8}\left(\mathrm{FeSe}_{0.98}\right)_{2}$, Phys. Rev. Lett. 106, 117602 (2011)
[16] X.-W. Yan, M. Gao, Z.-Y. Lu, and T. Xiang, Ternary Iron Selenide $\quad \mathrm{K}_{0.8} \mathrm{Fe}_{1.6} \mathrm{Se}_{2} \quad$ Is an Antiferromagnetic Semiconductor, Phys. Rev. B 83, 233205 (2011).

[17] C. Cao and J. Dai, Block Spin Ground State and 3-Dimensionality of (K, Tl) $\mathrm{Fe}_{1.6} \mathrm{Se}_{2}$, Phys. Rev. Lett. 107, 056401 (2011).

[18] C. Cao and J. Dai, Electronic Structure and Mott Localization of Iron-Deficient $\mathrm{TlFe}_{1.5} \mathrm{Se}_{2}$ with Superstructures, Phys. Rev. B 83, 193104 (2011).

[19] X.-W. Yan, M. Gao, Z.-Y. Lu, and T. Xiang, Electronic Structures and Magnetic Order of Ordered-Fe-Vacancy Ternary Iron Selenides $\mathrm{TlFe}_{1.5} \mathrm{Se}_{2}$ and $\mathrm{AFe}_{1.5} \mathrm{Se}_{2}$ $(\mathrm{A}=\mathrm{K}, R b$, or $C s)$, Phys. Rev. Lett. 106, 087005 (2011).

[20] See supplemental material at http://link.aps.org/ supplemental/10.1103/PhysRevX.1.021020 for the description of the out-of-plane momentum $\left(k_{z}\right)$ determination.

[21] T. Greber, T. J. Kreutz, and J. Osterwalder, Photoemission above the Fermi Level: The Top of the Minority d Band in Nickel, Phys. Rev. Lett. 79, 4465 (1997).

[22] B. Shen, B. Zeng, G. F. Chen, J. B. He, D. M. Wang, H. Yang, and H.H. Wen, Intrinsic Percolative Superconductivity in $\mathrm{K}_{x} \mathrm{Fe}_{2-y} \mathrm{Se}_{2}$ Single Crystals, Europhys. Lett. 96, 37010 (2011).

[23] D. A. Torchetti, M. Fu, D. C. Christensen, K. J. Nelson, T. Imai, H. C. Lei, and C. Petrovic, $\mathrm{S}^{77}$ e NMR Investigation of the High- $T_{c}$ Superconductor $\left(T_{c}=33 \mathrm{~K}\right)$, Phys. Rev. B 83, 104508 (2011).

[24] D. H. Ryan, W. N. Rowan-Weetaluktuk, J. M. Cadogan, R. Hu, W. E. Straszheim, S. L. Budko, and P. C. Canfield, 57Fe Mössbauer Study of Magnetic Ordering in Superconducting $\mathrm{K}_{0.85} \mathrm{Fe}_{1.83} \mathrm{Se}_{2.09}$ Single Crystals, Phys. Rev. B 83, 104526 (2011).

[25] J. Q. Li, Y. J. Song, H. X. Yang, Z. Wang, H. L. Shi, G. F. Chen, Z.W. Wang, Z. Chen, and H.F. Tian, Collapse of the Fe-Vacancy Order and Successive Phase Transitions in Superconducting $\mathrm{K}_{x} \mathrm{Fe}_{2-y} \mathrm{Se}_{2} \quad(0.7 \leq x \leq$ $0.8,0.2 \leq y \leq 0.3$ ), arXiv:1104.5340.

[26] C. Fang, B. Xu, P. Dai, T. Xiang, and J. Hu, Magnetic Frustration and Iron-Vacancy Ordering in IronChalcogenide, arXiv:1103.4599.

[27] Similar TEM images of the in-plane phase separation have been observed by Z. W. Wang, J. Q. Li, and their colleagues (private communications).

[28] See the numerical simulation presented in the supplementary material at http://link.aps.org/supplemental/10.1103/ PhysRevX.1.021020.

[29] R. H. Yuan, T. Dong, G. F. Chen, J. B. He, D. M. Wang, and N. L. Wang, Observation of a Small Superconducting Energy Gap in $\mathrm{K}_{0.7} \mathrm{Fe}_{1.8} \mathrm{Se}_{2}$ by Optical Spectroscopy, arXiv:1102.1381.

[30] H.-M. Jiang, W.-Q. Chen, Z.-J. Yao, and F.-C. Zhang, Superconducting Proximity Effect to the Block Antiferromagnetism in $\mathrm{K}_{x} \mathrm{Fe}_{2-y} \mathrm{Se}_{2}$, arXiv:1111.1860.

[31] W. Li, H. Ding, P. Deng, K. Chang, C. Song, K. He, L. Wang, X. Ma, J.-P. Hu, X. Chen, and Q.-K. Xue, Phase Separation and Magnetic Order in K-Doped Iron Selenide Superconductor, arXiv:1108.0069. 